\title{
MODELLING OF SIMULTANEOUS MASS AND HEAT TRANSFER WITH CHEMICAL REACTION USING THE MAXWELL-STEFAN THEORY-I. MODEL DEVELOPMENT AND ISOTHERMAL STUDY
}

\author{
M. J. W. FRANK ${ }^{\dagger}$, J. A. M. KUIPERS, G. F. VERSTEEG and W. P. M. VAN SWAAIJ \\ Department of Chemical Engineering, Twente University of Technology, P.O. Box 217, 7500 AE Enschede, \\ The Netherlands
}

(Received 28 July 1994; accepted in revised form 14 December 1994)

\begin{abstract}
A general applicable model has been developed which can predict mass and heat transfer fluxes through a vapour/gas-liquid interface in case a reversible chemical reaction with associated heat effect takes place in the liquid phase. In this model the Maxwell-Stefan theory has been used to describe the transport of mass and heat. The description of the transfer rates has been based on the film model in which a well-mixed bulk and a stagnant zone are thought to exist. In this paper results obtained from the Maxwell-Stefan theory have been compared with the results obtained from the classical theory due to Fick. This has been done for isothermal absorption of a pure gas $\mathrm{A}$ in a solvent containing a reactive component B. Component A is allowed to react by a unimolecular chemical reaction or by a bimolecular chemical reaction with B to produce component C. Since the Maxwell-Stefan theory leads to implicit expressions for the absorption rates, approximate explicit expressions have been derived. In case of absorption with chemical reaction it turned out that the mass transfer rate could be formulated as the product of the mass flux for physical absorption and an enhancement factor. This enhancement factor possesses the same functional dependency in case Fick's law is used to describe the mass transfer process. The model which has been developed in this work is quite general and can be used for a rather general class of gas-liquid and vapour-liquid transfer processes. In this paper (Part I) only isothermal simulations will be reported to show the important features of the model for describing mass transfer with chemical reaction. In many processes such as distillation, reactive distillation and some absorption processes, heat effects may play an important additional role. In Part II non-isothermal processes will be studied to investigate the influence of heat effects on mass transfer rates.
\end{abstract}

\section{INTRODUCTION}

Many industrial processes involve mass transfer processes between a gas/vapour and a liquid. Usually, these transfer processes are described on the basis of Fick's law, but the Maxwell-Stefan theory finds increasing application. Especially for reactive distillation it can be anticipated that the Maxwell-Stefan theory should be used for describing the mass transfer processes. Moreover, with reactive distillation there is a need to take heat transfer and chemical reaction into account. The model developed in this study will be formulated on a generalized basis and as a consequence it can be used for many other gas-liquid and vapour-liquid transfer processes. However, reactive distillation has recently received considerable attention in literature. With reactive distillation reaction and separation are carried out simultaneously in one apparatus, usually a distillation column. This kind of processing can be advantageous for equilibrium reactions. By removing one of the products from the reactive zone by evaporation, the equilibrium is shifted to the product side and consequently higher conversions can be obtained. Commercial applications of reactive distillation are the production of methyl-

'Author to whom correspondence should be addressed. acetate and the production of MTBE (Mikitenko, 1986; Agreda et al., 1990; Cieutat, 1989). A second reaction type of interest for reactive distillation is the consecutive reaction. By removing the desired product from the reactive zone, the undesired consecutive reaction can be suppressed resulting in improved process selectivity. However, little information is available in literature with respect to applications involving reactive distillation in case of consecutive reactions (Carrà et al., 1979; Corrigan and Miller, 1968).

Modelling of reactive distillation is usually based on the equilibrium stage-approach (Baratella et al., 1974; Nelson, 1971; Venkataraman et al., 1990). Similar to modelling of ordinary distillation processes, this approach will produce the required number of theoretical plates. For calculating the actual number of separation stages, stage efficiencies or HETPs are used in case of, respectively, a tray and a packed column. For binary distillation this method will give reliable results since both components possess the same efficiency which does not depend on the position in the column with values ranging from 0 to 1 . In case of multicomponent distillation each component will possess its own efficiency, which is a function of the position in the column with values which can range 
from $-\infty$ to $+\infty$. If chemical reaction occurs in addition a so-called reaction efficiency has to be introduced. It is clear that the equilibrium stage-concept will give rise to significant problems which necessitates another approach.

The variation of efficiencies is due to interaction phenomena caused by the simultaneous diffusional transport of several components. From a fundamental point of view one should therefore take these interaction phenomena explicitly into account in the description of the elementary processes (i.e. mass and heat transfer with chemical reaction). In literature this approach has been used within the non-equilibrium stage model (Sivasubramanian and Boston, 1990). Sawistowski (1983) and Sawistowski and Pilavakis (1979) have developed a model describing reactive distillation in a packed column. Their model incorporates a simple representation of the prevailing mass and heat transfer processes supplemented with a rate equation for chemical reaction, allowing chemical enhancement of mass transfer. They assumed elementary reaction kinetics, equal binary diffusion coefficients and equal molar latent heat of evaporation for each component.

In this paper a transfer model will be presented, which can predict mass and energy transport through a gas/vapour-liquid interface where a chemical reaction occurs simultaneously in the liquid phase. In this model the Maxwell-Stefan theory has been used to describe the transport of mass and heat. On the basis of this model a numerical study will be made to investigate the consequences of using the MaxwellStefan equation for describing mass transfer in case of physical absorption and in case of absorption with chemical reaction. Despite the fact that the MaxwellStefan theory has received significant attention, the incorporation of chemical reactions with associated heat effects has not yet been studied in great detail. Vanni and Baldi (1991) described mass transfer and chemical reaction using the aforementioned theory, but they did not take heat transport into account. Besides, they made specific assumptions with respect to the volatility of components and reaction stoichiometry and kinetics. Additional work is available in literature (Mann and Moyes, 1977; Chatterjee and Altwicker, 1987; Al-Ubaidi et al., 1990; Bhattacharya et al., 1987) dealing with mass and heat transfer processes with simultaneous chemical conversion; however, the theory of multicomponent diffusion has not been used in these studies.

\section{THEORY}

The mass and heat transport model should be able to predict mass and energy fluxes through a gas/vapour-liquid interface in case a chemical reaction occurs in the liquid phase. In this study the film model will be adopted which postulates the existence of a well-mixed bulk and a stagnant transfer zone near the interface (see Fig. 1). The equations describing the mass and heat fluxes play an important role in our model and will be presented subsequently.

Since interaction phenomena due to simultaneous diffusion of several components play an important role, the Maxwell-Stefan theory has been selected to describe the mass transfer processes. The general form of the flux expressions can be represented by (Taylor and Krishna, 1993)

$$
r_{i}=d_{i}
$$

where the driving forces $d_{i}$ acting on a component $i$ are set equal to the resistance forces $r_{i}$ experienced by the same component. From irreversible thermodynamics the following expressions for the forces are

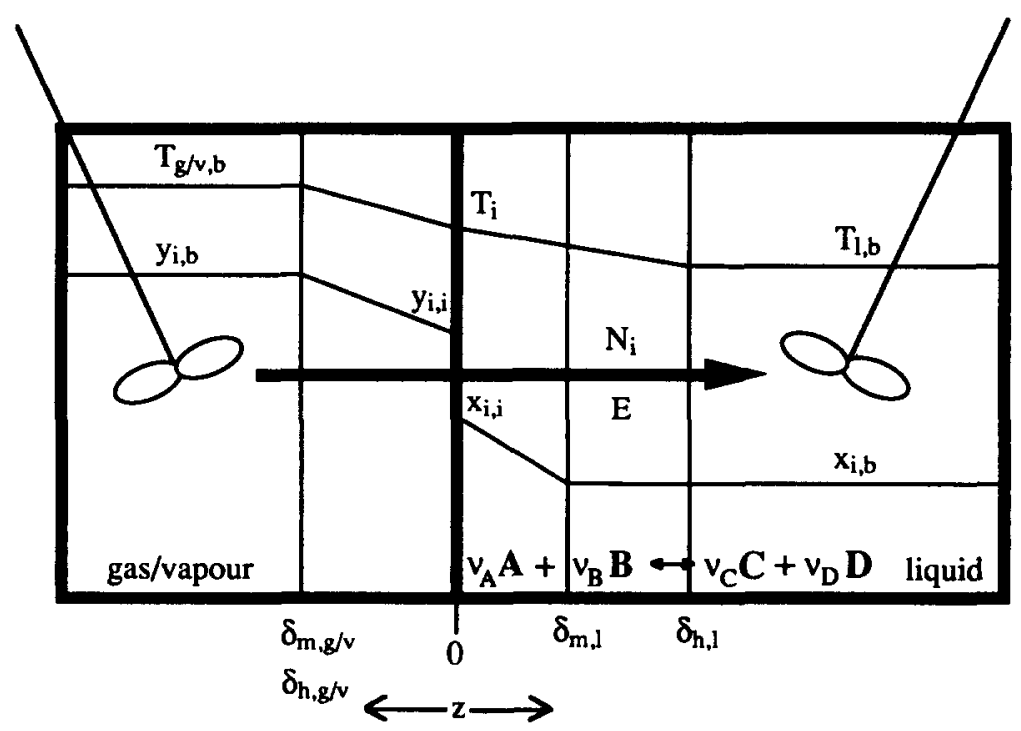

Fig. 1. Schematic representation of the region near the gas/vapour-liquid interface in terms of the film model. 
obtained:

$$
\begin{array}{r}
r_{i}=-\sum_{\substack{j=1 \\
i \neq i}}^{n} \frac{x_{i} x_{j}\left(u_{i}-u_{j}\right)}{D_{i j}}-\sum_{\substack{j=1 \\
i \neq i}}^{n} x_{i} x_{j} \alpha_{i j} \frac{\nabla T}{T} \\
c_{T} R_{\mathrm{gas}} T d_{i}=c_{i}\left(\nabla \mu_{i}\right)_{P, T}+\left(\phi_{i}-\omega_{i}\right) \nabla p \\
-\left(c_{i} F_{i}-\omega_{i} \sum_{j=1}^{n} c_{j} F_{j}\right) .
\end{array}
$$

The first resistance term is due to binary diffusional interactions and the second term accounts for the Soret effect. The first term on the right-hand side of eq. (3) represents the gradient of the chemical potential of component $i$ at constant temperature and pressure. The second term is due to the pressure gradient, which only plays a role, when the weight fraction and volume fraction differ. The last term represents a general expression for a driving force and can be used, for example, to represent body forces and forces due to imposed electric fields. In our model it has been assumed that the driving force is completely determined by the gradient of the chemical potential and additionally that the Soret effect is of no importance. Assuming a thermodynamic ideal system, the following dimensionless representation of the Maxwell -Stefan flux equations can be obtained:

$$
\frac{\mathrm{d} x_{i}}{\mathrm{~d} \eta}=\sum_{\substack{j=1 \\ j \neq 1}}^{j=n} \frac{x_{i} N_{j}-x_{j} N_{i}}{c_{T} K_{i j}}
$$

where $\eta$ represents a dimensionless coordinate, $K_{i j}$ a binary mass transfer coefficient, $c_{T}$ the total molar concentration and $N_{j}$ the molar flux of component $j$ with respect to a stationary reference frame.

The energy flux consists of a conductive and a convective contribution. The general expression for the conductive flux is given by

$$
q=-\lambda \nabla T+\frac{1}{2} c_{T} R_{\mathrm{gas}} T \sum_{i=1}^{n} \sum_{\substack{j=1 \\ j \neq 1}}^{n} x_{i} x_{j} \alpha_{i j}\left(u_{i}-u_{j}\right)
$$

The first term on the right-hand side of eq. (5) describes the heat conduction and the second term accounts for the Dufour effect. The convective contribution of the energy flux is given by

$$
E_{\text {conv. }}=\sum_{i=1}^{i=n} N_{i} H_{i}
$$

where the partial molar enthalpy $H_{i}$ is expressed by a caloric equation of state with constant $C_{p i}$ :

$$
H_{i}=H_{i, r_{\text {rer. }}}+C_{p i}\left(T-T_{\text {ref. }}\right) \text {. }
$$

The heat of vaporization or the heat of dissolution has been incorporated in the term $H_{i, T_{\text {ret }}}$. Neglecting the Dufour effect, the total energy flux $E$ is given by

$$
E=\sum_{i=1}^{i=n} N_{i} H_{i}-h L e^{\prime} \frac{\mathrm{d} T}{\mathrm{~d} \eta}
$$

where $h$ represents a heat transfer coefficient, $\eta$ a dimensionless distance and $L e^{\prime}$ a modified Lewis number defined as $\delta_{n} / \delta_{m}$. As stated earlier the film model has been adopted in the present study which requires specification of the film thickness for both the mass and heat transfer film. In the gas/vapour phase it has been assumed that the thickness of both films is the same which is justified since the Lewis number approximately equals one in this case. In the liquid phase, however, the thickness of the heat transfer film can exceed the thickness of the mass transfer film considerably. This has been taken into account in our model. Consequently, beyond the mass transfer boundary it has been assumed that the convective and the conductive contribution of the energy transport are decoupled since two different transport mechanisms are involved (respectively, mixing and conduction). The convective contribution will directly enter the liquid bulk, whereas the conductive contribution $E_{c}$ has to obey eq. (9) from $z=\delta_{m, l}$ to $z=\delta_{h, l}$ before it reaches the liquid bulk:

$$
E_{c}=-h L e^{\prime} \frac{\mathrm{d} T}{d \eta} .
$$

In addition to the expression for the mass and energy fluxes, conservation equations for mass and energy are required to enable the calculation of concentration and temperature profiles. From these profiles the mass and heat transfer rates through the vapour/gas-liquid interface can subsequently be obtained. The species conservation equations for the liquid and the vapour/gas phase are respectively given by

$$
\frac{\mathrm{d} N_{i}}{\mathrm{~d} \eta}-v_{i} R \delta_{m}=0
$$

and

$$
\frac{\mathrm{d} N_{i}}{\mathrm{~d} \eta}=0 .
$$

Note that the mass flux of component $i$ in the liquid phase changes due to chemical conversion, whereas this flux remains constant in the vapour/gas phase since it has been assumed that no reaction occurs in the vapour/gas phase. For both phases the conservation for thermal energy equation is given by

$$
\frac{\mathrm{d} E}{\mathrm{~d} \eta}=0 .
$$

Since partial molar enthalpies are used in the energy flux description (6), no source term appears in eq. (12).

The following chemical reaction is assumed to take place in the liquid:

$$
v_{a} \mathrm{~A}+v_{b} \mathrm{~B} \leftrightarrow v_{c} \mathrm{C}+v_{d} \mathrm{D}
$$

where the reaction rate is expressed by the following power law formulation in which the temperature dependency of the kinetic constants is represented by an Arrhenius-type expression:

$$
\begin{aligned}
R= & k_{01} \exp \left(-\frac{E_{a 1}}{R_{\text {gas }} T}\right) x_{A}^{a} x_{B}^{b} \\
& -k_{02} \exp \left(-\frac{E_{a 2}}{R_{\text {gas }} T}\right) x_{C}^{c} x_{D}^{d} .
\end{aligned}
$$


To obtain an unique solution of the differential equations boundary conditions are required. The following boundary conditions have been used in this study (see Fig. 1):

-at gas/vapour bulk-film interface $\left(z=\delta_{m, g / v}\right.$ $\left.=\delta_{h, g / v}\right)$ :

$$
y_{i}=y_{i, b}
$$

and

$$
T=T_{g / v, b}
$$

-at gas/vapour-liquid interface $(z=0)$ :

$$
y_{i}=\frac{p_{g / v, i}(T)}{p} x_{i}
$$

-at liquid bulk-mass transfer film interface $\left(z=\delta_{m, l}\right)$ :

$$
x_{i}=x_{i, b}
$$

and

$$
E_{c}=q_{\text {mass }} \text { transfer film }
$$

-at liquid bulk-heat transfer film interface $\left(z=\delta_{h, l}\right)$ :

$$
T=T_{l, b} \text {. }
$$

Conditions (15a), (15b), (15d) and (15f) result from the imposed bulk conditions. Condition (15c) describes the gas/vapour-liquid equilibrium at the gas/vapour-liquid interface where the gas/vapour pressure can be represented by

$$
p_{g / v, i}=\exp \left(A_{1}-\frac{A_{2}}{A_{3}+T}\right)
$$

or

$$
p_{g / v, i}=\frac{p}{m_{i}} \exp \left[\frac{-E_{s}}{R_{\mathrm{gas}}}\left(\frac{1}{T}-\frac{1}{T_{0}}\right)\right] .
$$

The Antoine equation (16a) is normally used for a vapour-liquid mixture, whereas eq. (16b) is normally used for gas-liquid mixtures. Condition (15e) states that the conductive contribution of the energy flux which reaches the boundary of the liquid mass transfer film is passed through to the heat transfer film obeying eq. (9). The convective contribution of the energy transport enters the liquid bulk independently.

In the gas/vapour phase the dimensionless distance $\eta$ ranges from 0 to 1 , where $\eta=1$ corresponds to the position of the interface. In the liquid phase this parameter ranges from 0 to 1 for the mass transfer film and from 0 to $L e^{\prime}$ for the heat transfer film. Hence, $\eta=0$ corresponds to the position of the interface and $\eta=1$ and $\eta=L e^{\prime}$ correspond, respectively, to the boundaries of the mass and heat transfer film. The mass and energy fluxes can now be calculated by solving the differential equations (4) and (8)-(12) subject to the boundary conditions (15). Due to the non-linearities a numerical solution procedure has been used which will be discussed subsequently.

\section{NUMERICAL SOLUTION METHOD}

The differential equations were solved numerically by a finite-difference method. The resulting system of non-linear equations were solved with the multidimensional Newton-Raphson method. Figure 2 schematically shows the finite-difference representation of the computational domain. On both sides of the gas/vapour-liquid interface the dimensionless distance $\eta$ has been subdivided into segments of equal length resulting in an equidistant distribution of the grid points. No grid points were distributed beyond the boundary of the mass and heat transfer film (i.e. for $k>K_{1}+K_{2}$ ) since in this part of the domain differential equation (9) with boundary condition (15f) can be solved analytically. This yields a new boundary condition which has to be satisfied at the boundary of the mass and heat transfer film:

$$
T_{\eta=1}=T_{l, b}+\frac{E_{c}}{h_{l}}\left(1-\frac{1}{L e^{\prime}}\right)
$$

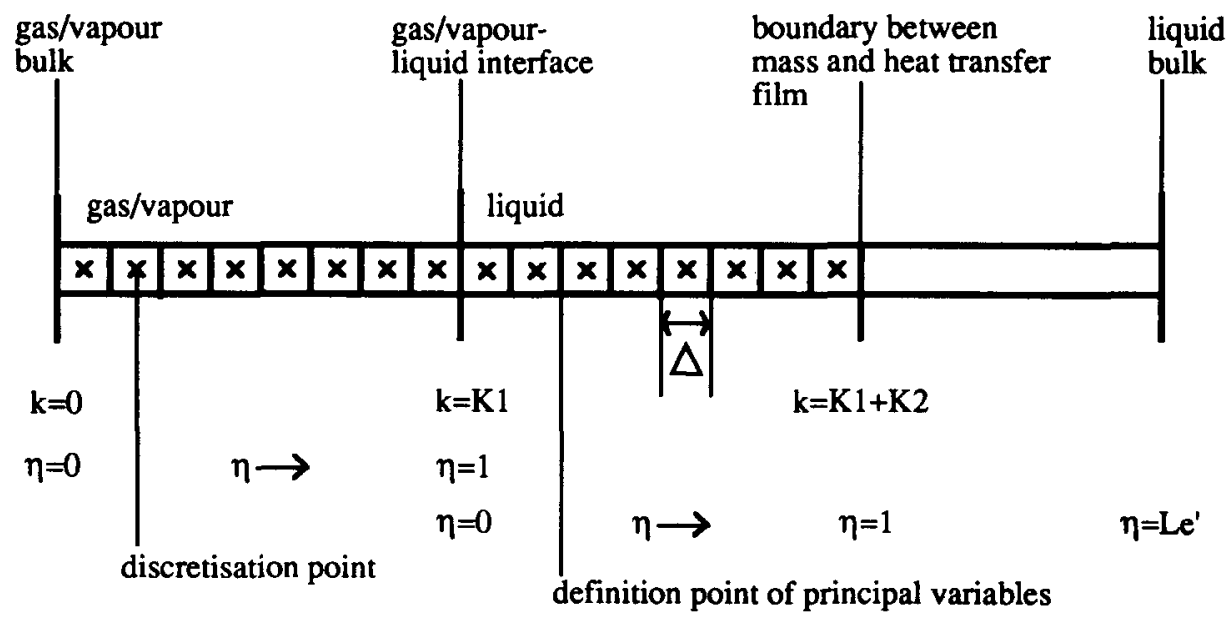

Fig. 2. Schematic representation of the computational domain employed in the finite-difference technique. 
where $E_{c}$ represents the energy flux prevailing in the heat transfer film, which has to obey boundary condition (15e).

The discretization points and the variables have been defined at, respectively, the centre of the segment and the boundary between two neighbour segments. Standard second-order finite difference approximations were used to discretize the spatial derivatives:

$$
\left(\frac{\mathrm{d} Y}{\mathrm{~d} \eta}\right)_{k-1 / 2}=\frac{Y_{k}-Y_{k-1}}{\Delta}+O\left(\Delta^{2}\right)
$$

in which $Y$ represents a principal variable. Simple linear interpolations were used whenever variables were required at other positions than defined according to Fig. 2:

$$
Y_{k-1 / 2}=\frac{Y_{k}+Y_{k-1}}{2}
$$

To enable solution of the set of equations, the number of unknown variables and the number of (non-linear) equations should be equal. The variables are $x_{i}\left(y_{i}\right)$, $N_{i}, T$ and $E$, so in case of $n$ components and $K_{1}+K_{2}$ discretization points we have $\left(K_{1}+K_{2}+1\right)(2 n+2)$ unknowns. At each discretization point, eq. (4) has been used for components 1 to $n-1$, whereas for component $n$ this equation has been replaced by

$$
\sum_{i=1}^{i=n} x_{i}=1 .
$$

Discretization of eqs (10) and (11) for, respectively, the liquid phase and the gas/vapour phase together with the discretization of the conservation equation for thermal energy (12) and the energy flux equation (8) leads to a total of $\left(K_{1}+K_{2}\right)(2 n+2)$ non-linear equations. Boundary conditions (15a), (15b), (15d) and (17) provide the remaining $(2 n+2)$ relations.

The system of non-linear equations has been solved by the multidimensional Newton-Raphson technique which involves the successive solution of a linear system of equations:

$$
A^{j \cdot} \bar{y}^{j+1}=\bar{B}^{j} \quad(j=0,1, \ldots)
$$

where $A^{j}$ represents the Jacobi matrix, $\bar{y}^{j+1}$ the vector of all unknown variables and $\bar{B}^{j}$ the residue vector. The linearized set of equations has been solved using a LU-decomposition technique. All calculations reported in this paper were performed on a personal computer.

\section{ISOTHERMAL RESULTS}

The model described in the previous sections is very complicated and therefore in Part I only isothermal studies will be reported. This limiting situation can be obtained by putting $H_{i}=0(i=1, \ldots, n)$ and setting the bulk temperatures equal to $T_{0}$ in eq. (16b). In this paper the influence of multicomponent transport phenomena on the mass transfer rate between a gas/vapour and a liquid will be studied in detail for both non-reactive and reactive conditions. It should be stressed that the validity of the model developed in this study is not limited to the low concentration domain. Subsequently, a number of isothermal mass transfer calculations will be reported with increasing degree of complexity.

\subsection{Mass transfer without chemical reaction}

In this case it is assumed that a pure gas A is being absorbed in a solvent containing a chemically inert component B. Both the solvent and B are not volatile and the fraction of $A$ in the liquid bulk equals zero. The binary mass transfer coefficient $K_{i j}$ between $A$ and the solvent in eq. (4) is given a typical value of $1 \times 10^{-4} \mathrm{~m} / \mathrm{s}$, whereas the total concentration of the liquid $c_{T}$ is set to $1 \times 10^{4} \mathrm{~mol} / \mathrm{m}^{3}$, also a typical value. Parameters to be chosen are: the solubility of $\mathrm{A}, x_{A i}$, the fraction of $\mathrm{B}$ in the solvent $x_{B b}$, the mass transfer coefficient between $\mathrm{A}$ and $\mathrm{B}, K_{\mathrm{AB}}$ and the mass transfer coefficient between $\mathrm{B}$ and the solvent, $K_{B s}$. The results of the calculations are presented in Table 1. Since both the solvent and component $B$ possess a zero flux, $K_{B s}$ has no influence on the mass transfer process and has therefore been omitted. The computed absorption rate has been compared with the absorption rate obtained from analytical solutions for the following cases.

(I) Mass transfer is described by Fick's law with superimposed drift:

$$
n_{A}=-\ln \left(1-x_{A i}\right) .
$$

(II) Mass transfer is described by the Maxwell-Stefan theory (the algebraic derivation is given in Appendix A):

$$
\begin{aligned}
1- & \left(1-x_{A i}\right) \exp \left(n_{A}\right) \\
& +x_{B b}\left\{\exp \left[n_{A}\left(1-d_{A B}\right)\right]-1\right\}=0
\end{aligned}
$$

where $d_{A B}$ denotes the ratio of the diffusion coefficient of $A$ in the solvent and the diffusion coefficient of $A$ in B $\left(d_{A B}=D_{A s} / D_{A B}\right)$.

(III) Mass transfer is described by a modified Maxwell-Stefan theory by assuming $n_{A} \ll 1$ and $d_{A B} n_{A} \ll 1$ (the absorption flux is sufficiently small). In this case eq. (22) can be reduced to the following explicit expression for the dimensionless absorption flux by linearization of the exponential terms (see Appendix A):

$$
n_{A}=\frac{x_{A i}}{1+x_{B b}\left(d_{A B}-1\right)-x_{A i}} .
$$

(IV) Mass transfer is described by a modified Maxwell-Stefan theory where expression (23) is modified in such a way that it reduces to eq. (21) in case $d_{A B}=1$ :

$$
n_{A}=\frac{-\ln \left(1-x_{A i}\right)}{1+x_{B b}\left(d_{A B}-1\right)} .
$$

In all cases $n_{A}$ represents a dimensionless absorption flux $\left(n_{A}=N_{A} / K_{A s} c_{T}\right)$. The explicit expression for $n_{A}$ given in case (III) is the same as eq. (24) from Vanni and Baldi (1991) by taking, following their nomenclature, $H a=0, x_{3}=0, x_{4}=1-x_{2 \delta}$ and $x_{10} \ll 1$. The 
Table 1. Dimensionless mass flux $n_{A}$ for mass transfer without chemical reaction. [The results obtained from eqs (21), (23) and (24) are compared with the results obtained from the numerical model]

\begin{tabular}{|c|c|c|c|c|c|c|c|}
\hline \multirow[t]{2}{*}{ Run } & \multirow[t]{2}{*}{$x_{A i}$} & \multirow[t]{2}{*}{$x_{B b}$} & \multirow[t]{2}{*}{$d_{A B}$} & \multirow{2}{*}{$\begin{array}{c}n_{A} \\
\text { model }\end{array}$} & \multicolumn{3}{|c|}{ Relative deviation (\%) } \\
\hline & & & & & eq. (21) & eq. (23) & eq. (24) \\
\hline 1 & 0.001 & 0.001 & 1 & 0.0010 & 0 & 0.1 & 0 \\
\hline 2 & 0.001 & 0.5 & 1 & 0.0010 & 0 & 0.1 & 0 \\
\hline 3 & 0.1 & 0.001 & 1 & 0.1054 & 0 & 5 & 0 \\
\hline 4 & 0.1 & 0.5 & 1 & 0.1054 & 0 & 5 & 0 \\
\hline 5 & 0.5 & 0.001 & 1 & 0.6929 & 0 & 44 & 0 \\
\hline 6 & 0.5 & 0.5 & 1 & 0.6929 & 0 & 44 & 0 \\
\hline 7 & 0.001 & 0.001 & 2 & 0.0010 & 0.1 & 0.1 & 0 \\
\hline 8 & 0.001 & 0.5 & 2 & 0.0007 & 50 & 0 & 0 \\
\hline 9 & 0.1 & 0.001 & 2 & 0.1053 & 0.1 & 5 & 0 \\
\hline 10 & 0.1 & 0.5 & 2 & 0.0707 & 49 & 1 & -0.6 \\
\hline 11 & 0.5 & 0.001 & 2 & 0.6924 & 0.1 & 44 & 0 \\
\hline 12 & 0.5 & 0.5 & 2 & 0.4810 & 44 & 4 & -4 \\
\hline 13 & 0.001 & 0.001 & 0.5 & 0.0010 & -0.1 & 0.1 & 0 \\
\hline 14 & 0.001 & 0.5 & 0.5 & 0.0013 & -25 & 0.1 & 0 \\
\hline 15 & 0.1 & 0.001 & 0.5 & 0.1054 & -0.1 & 5 & 0 \\
\hline 16 & 0.1 & 0.5 & 0.5 & 0.1413 & -25 & 9 & -0.6 \\
\hline 17 & 0.5 & 0.001 & 0.5 & 0.6933 & -0.1 & 44 & 0 \\
\hline 18 & 0.5 & 0.5 & 0.5 & 0.9619 & -28 & 108 & -4 \\
\hline
\end{tabular}

difference between the numerical results and the results obtained from analytical solution (22) is always smaller than $0.04 \%$ indicating the accuracy of the numerical calculations. According to Table 1 in case of equal diffusivities, eq. (21) can be used to estimate the mass flux of A, regardless the value of the solubility. In systems where the diffusivities differ and where small fractions of $\mathrm{B}$ in the solvent prevail, the error is typically smaller than $0.1 \%$. For large values of $x_{B b}$, eq. (21) cannot be used anymore. The explicit expression (23) can be used if the solubility of $A$ in the solvent is sufficiently low where the error does not depend much on the fraction of $B$. The modified explicit expression (24) produces the smallest errors and can be used over a very wide range of solubility of $A$, bulk fraction of $B$ and ratio of diffusion coefficients of $A$ and $B$ in the solvent. The maximum error produced by eq. (24) as shown in Table 1 is approximately $4 \%$.

Figures 3(a) and 3(b) show the computed fraction profiles of component $A$ and $B$ in the liquid film corresponding to, respectively, run 1 and run 6 from Table 1. Figure 3(a) shows that low fractions of $A$ and B produce straight profiles, whereas high fractions of $A$ and $B$ result in curved profiles [see Fig. 3(b)]. The latter is due to the fact that the mass fluxes consist of a diffusive part as well as a convective (i.e. drift) part. This is also the reason why the fraction of $B$ possesses a gradient, although the flux of component $B$ equals zero.

\subsection{Mass transfer with instantaneous chemical reaction}

In this case a pure gas $A$ is assumed to absorb in a solvent containing a reactive component $B$ where the following chemical reaction takes place in the
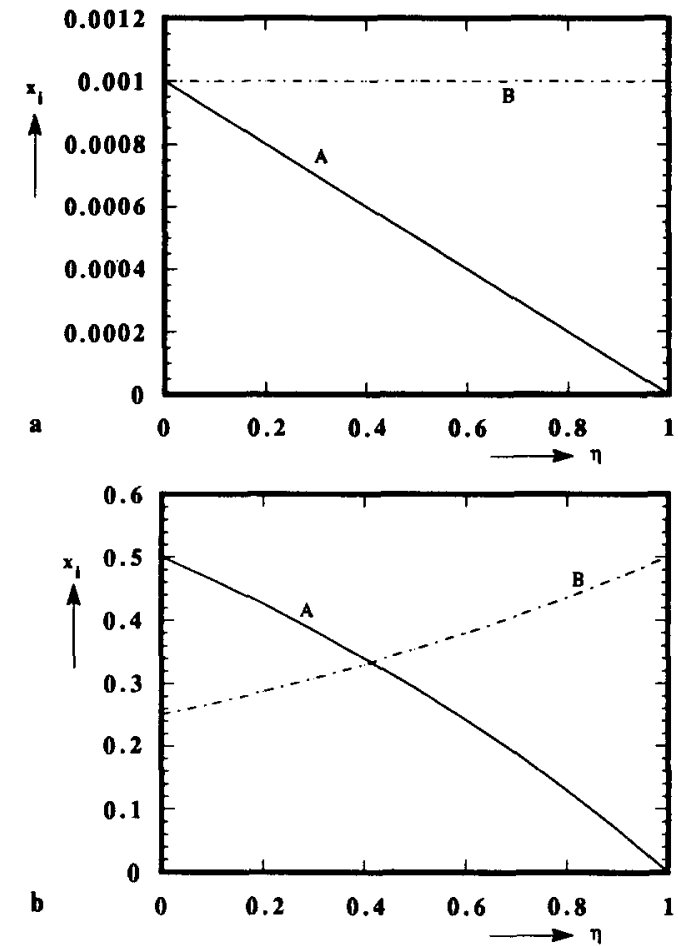

Fig. 3. Computed fraction profiles of components $A$ and $B$ in the liquid film corresponding to (a) run 1 and (b) run 6 from Table 1 for mass transfer without chemical reaction.

solvent:

$$
\mathrm{A}+\mathrm{B} \rightarrow \mathrm{C}
$$

For this situation an irreversible chemical reaction with first-order kinetics with respect to $A$ and $B$ has been used, where a very high value of the reaction rate constant has been taken to simulate an instantaneous 
reaction. The solvent, component $B$ and product $\mathrm{C}$ are not volatile and the fractions of $\mathrm{A}$ and $\mathrm{C}$ in the liquid bulk equal zero. The mass transfer coefficient $K_{i j}$ between $A$ and the solvent in eq. (4) is given a typical value of $1 \times 10^{-4} \mathrm{~m} / \mathrm{s}$, whereas the total concentration of the liquid $c_{T}$ is set to $1 \times 10^{4} \mathrm{~mol} / \mathrm{m}^{3}$, also a typical value. Parameters to be chosen are: the solubility of $A, x_{A i}$, the fraction of $\mathrm{B}$ in the solvent $x_{B b}$ and the mass transfer coefficients $K_{B s}, K_{C s}, K_{A B}$, $K_{A C}$ and $K_{B C}$. The results of the model calculations are given in Table 2. Figures $4(a)$ and $4(b)$ show the computed fraction profiles of component $\mathrm{A}, \mathrm{B}$ and $\mathrm{C}$ in the liquid film corresponding to, respectively, run 1 and run 2 from Table 2. Figure 4(a) shows that low fractions of $A$ and $B$ produce straight profiles, whereas high fractions of $A$ and $B$ result in slightly curved profiles and a maximum in the fraction profile of component $C$ [see Fig. 4(b)]. This phenomenon is due to the fact that the fluxes consist of a diffusive part as well as a convective part. This leads to a gradient in the fraction of $\mathrm{C}$ at the left side of the reaction plane, although the flux of component $\mathrm{C}$ equals zero in this part of the film. The computed absorption rate has been compared with the absorption rates obtained from analytical solutions for a number of limiting cases which will be discussed subsequently.

(I) Mass transfer is described by Fick's law:

$$
n_{A}=\left(1+\frac{x_{B b}}{d_{B S} x_{A i}}\right) x_{A i}
$$

where $d_{B s}$ represents the ratio of the binary diffusion coefficients: $d_{B s}=D_{A s} / D_{B s}$.

(II) Mass transfer is described by the Maxwell-Stefan theory [eq. (4)]. In this case the dimensionless absorption rate $n_{A}$ and the dimensionless coordinate of the reaction plane $f$ are given implicitly by the following two equations (an algebraic deriva- tion is given in Appendix B):

$$
\begin{gathered}
\left(x_{A i}-1\right) \exp \left(n_{A} f\right)+\exp \left[\left(1-d_{A C}\right) n_{A} f\right] \\
-\left(1-x_{B b}\right)\left\{\exp \left[\left(1-d_{A C}\right) n_{A} f\right]-1\right\} \\
\quad \times \exp \left[\left(d_{B s}-d_{C s}\right) n_{A}(1-f)\right]=0 \\
x_{B b}-d_{B C} n_{A}(1-f)+\frac{d_{B s}-d_{B C}}{d_{B s}-d_{C s}}\left(1-x_{B b}\right) \\
\quad \times\left\{1-\exp \left[\left(d_{B s}-d_{C s}\right) n_{A}(1-f)\right]\right\}=0
\end{gathered}
$$

where $d_{A C}, d_{B C}$ and $d_{C s}$ represent, respectively, the following ratios of the binary diffusion coefficients $D_{A s} / D_{A C}, D_{A s} / D_{B C}$ and $D_{A s} / D_{C s}$.

(III) Mass transfer is described by the Maxwell-Stefan theory. In addition, the following assumptions have been made which allow a drastic simplification of the implicit expressions (26a) and (26b).

(a) $1-d_{A C}=0$ and $d_{B s}-d_{C s}=0$ : In this case the diffusion coefficients of $A$ in the solvent and $A$ in $C$ are equal and in addition the diffusion coefficients of $B$ and $C$ in the solvent are equal. Substitution of $1-d_{A C}=0$ and $d_{B s}-d_{C s}=0$ in eq. (26) leads to

$$
n_{A}=\frac{x_{B b}}{\left(d_{B C}-d_{B s}\right) x_{B b}+d_{B s}}-\ln \left(1-x_{A i}\right)
$$

(b) $1-d_{A C}=0$ and $d_{B s}-d_{C s} \neq 0$ : In this case the diffusion coefficients of $A$ in the solvent and $A$ in $C$ are equal; however, the diffusion coefficients of $B$ and $C$ in the solvent differ. With the additional assumption $\left|\left(d_{B s}-d_{C s}\right)(1-f) n_{A}\right| \ll 1$, which allows linearization of the remaining exponential terms (see Appendix A), this will also lead to eq. (27).

\begin{tabular}{|c|c|c|c|c|c|c|c|}
\hline \multirow[t]{2}{*}{ Run } & \multirow[t]{2}{*}{$x_{A i}$} & \multirow[t]{2}{*}{$x_{B b}$} & \multirow{2}{*}{$\begin{array}{c}\text { Change with respect } \\
\text { to the } \\
\text { standard case }\end{array}$} & \multirow{2}{*}{$\begin{array}{c}n_{A} \\
\text { eq. (26) }\end{array}$} & \multicolumn{3}{|c|}{ Relative deviation $(\%)$} \\
\hline & & & & & Model & eq. (25) & eq. (30) \\
\hline 1 & 0.001 & 0.002 & - & 0.003 & 0 & 0 & 0 \\
\hline 2 & 0.3 & 0.6 & - & 0.957 & -0.4 & -6 & 0 \\
\hline 3 & 0.001 & 0.002 & $d_{B s}=2$ & 0.002 & 0 & 0 & 0 \\
\hline 4 & 0.3 & 0.6 & $d_{B s}=2$ & 0.759 & -0.3 & -21 & 3.6 \\
\hline 5 & 0.001 & 0.002 & $d_{c s}=2$ & 0.003 & 0 & 0 & 0 \\
\hline 6 & 0.3 & 0.6 & $d_{c s}=2$ & 0.957 & -0.4 & -6 & 0 \\
\hline 7 & 0.3 & 0.6 & $d_{A B}=2$ & 0.957 & -0.9 & -6 & 0 \\
\hline 8 & 0.3 & 0.6 & $d_{A C}=2$ & 0.827 & 0.1 & 9 & -0.5 \\
\hline 9 & 0.3 & 0.6 & $d_{B C}=2$ & 0.732 & -0.4 & 23 & 0 \\
\hline 10 & 0.3 & 0.6 & $d_{B s}=0.5$ & 1.078 & -0.3 & 39 & 2.7 \\
\hline 11 & 0.3 & 0.6 & $d_{C s}=0.5$ & 0.957 & -0.4 & -6 & 0 \\
\hline 12 & 0.3 & 0.6 & $d_{A B}=0.5$ & 0.957 & 0.2 & -6 & 0 \\
\hline 13 & 0.3 & 0.6 & $d_{A C}=0.5$ & 1.121 & -1.6 & -20 & -1 \\
\hline 14 & 0.3 & 0.6 & $d_{B C}=0.5$ & 1.214 & -0.2 & -26 & 0 \\
\hline
\end{tabular}

(c) $1-d_{A C} \neq 0$ and $d_{B s}-d_{C s}=0$ : In this case the diffusion coefficients of $A$ in the solvent and $A$ in $C$ differ; however, the diffusion coefficients of $B$ and

Table 2. Dimensionless mass flux $n_{A}$ for mass transfer with instantaneous chemical reaction. [The results obtained from the numerical model and eqs (25) and (30) are compared with the results obtained from the analytical solution, eq. (26)]

Standard case: $K_{A s}=1 \times 10^{-4} \mathrm{~m} / \mathrm{s} ; d_{A B}=d_{A C}=d_{B C}=d_{B s}=d_{C s}=1$ 


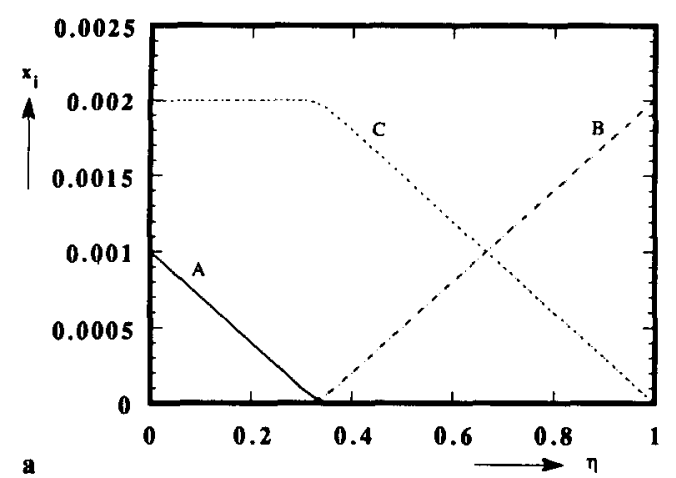

a

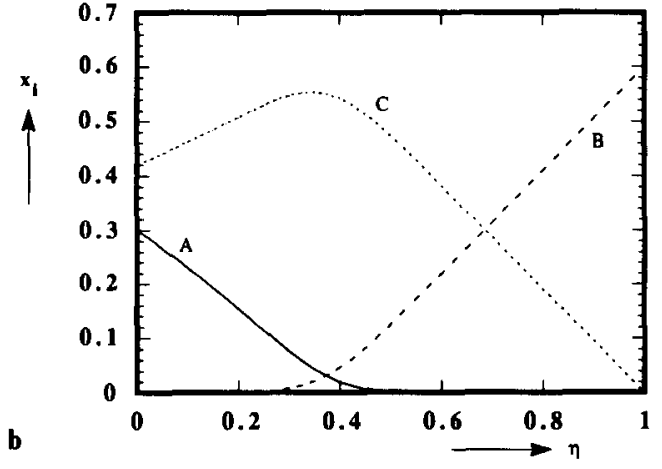

Fig. 4. Computed fraction profiles of components A, B and $C$ in the liquid film corresponding to (a) run 1 and (b) run 2 from Table 2 for mass transfer with instantaneous bimolecular chemical reaction.

$\mathrm{C}$ in the solvent are equal. With the additional assumptions $f n_{A} \ll 1$ and $d_{A C} f n_{A} \ll 1$, eq. (26) reduces to

$$
n_{A}=\frac{x_{B b}}{\left(d_{B C}-d_{B s}\right) x_{B b}+d_{B s}}+\frac{x_{A i}}{\left(d_{A C}-1\right) x_{B b}+1-x_{A i}} .
$$

(d) $1-d_{A C} \neq 0$ and $d_{B s}-d_{C s} \neq 0$ : In this case the diffusion coefficients of $A$ in the solvent and $A$ in $\mathrm{C}$ differ and in addition the diffusion coefficients of $B$ and $C$ in the solvent are different. With the additional assumptions $f n_{A} \ll 1, \quad d_{A C} f n_{A} \ll 1$ and $\left|\left(d_{B s}-d_{C s}\right)(1-f) n_{A}\right| \ll 1$ the resulting expression is given by

$$
\begin{aligned}
n_{A}= & \frac{x_{B b}}{\left(d_{B C}-d_{B s}\right) x_{B b}+d_{B s}} \\
& +\frac{x_{A i}}{\left(d_{A C}-1\right) x_{B b} \frac{d_{C s}+\left(d_{B C}-d_{C s}\right) x_{B b}}{d_{B s}+\left(d_{B C}-d_{B s}\right) x_{B b}}+1-x_{A i}}
\end{aligned}
$$

On the basis of inspection of eqs (27)-(29) and combination of the results for cases (a)-(d) the following general approximate explicit expression for the dimensionless absorption flux can be formulated:

$$
\begin{aligned}
n_{A}= & \frac{x_{B b}}{\left(d_{B C}-d_{B s}\right) x_{B b}+d_{B s}} \\
& -\frac{\ln \left(1-x_{A i}\right)}{\left(d_{A C}-1\right) x_{B b} \frac{d_{C s}+\left(d_{B C}-d_{C s}\right) x_{B b}}{d_{B s}+\left(d_{B C}-d_{B s}\right) x_{B b}}+1}
\end{aligned}
$$

Equation (30) reduces to eq. (27) in case $d_{A C}=1$, to eq. (28) in case $d_{B s}=d_{C s}$ and small $x_{A i}$ and to eq. (29) in case of small $x_{A i}$. Equation (30) has been derived from eq. (26) by making a first-order expansion of the exponential terms. Higher-order expansions are of course possible and will be more accurate, but will also lead to more complex explicit functions. The explicit expression for $n_{A}$ given in case (IIIc) is the same as eq. (57) from Vanni and Baldi (1991) by taking, following their nomenclature, $v_{2}=v_{3}=1$, $x_{3}=0, x_{4}=1-x_{2 \delta}$ and $x_{10} \ll 1$. According to Table 2 the difference between the numerical results and the results obtained from analytical solution (26) is always smaller than $1.6 \%$. This remaining difference is probably due to the value of the reaction rate constant which might have been chosen too low to simulate an instantaneous reaction perfectly. Due to anticipated problems with numerical stability it was not attempted to use higher values of the reaction rate constant. As shown in Table 2, Fick's law, eq. (25), produces errors up to $40 \%$ and can therefore only be used when the fractions of A and B are very small. The explicit expression (30) has also been compared with the results obtained from eq. (26) and it was found that the maximum difference amounted to $3.6 \%$.

Equations (25) and (30) have also been tested under more severe conditions. The numerical data used in the simulations are shown in Table 3. Figure 5(a) shows the results obtained from eq. (25) vs the results obtained from the exact analytical solution [eq. (26)]. Figure 5(b) shows the results obtained from eq. (30) vs the results obtained from the exact analytical solution [eq. (26)]. From Fig. 5(a) it can be seen that the mass fluxes cannot be predicted on the basis of Fick's law. It turned out that eq. (30) could predict the mass flux in $75 \%$ of the situations resulting in an error typically less than 5\%. By examining other situations and making additional calculations it turned out that errors exceeding 5\% occurred in the following cases:

$-d_{B C} \approx d_{B s} \ll d_{C s}\left(D_{B C} \approx D_{B s} \gg D_{C s}\right) \quad$ and $\quad d_{A C} \ll$ $d_{C s}\left(D_{A C} \gg D_{C s}\right)$ and $x_{A i} \geqslant 0.05$ and $x_{B b} \geqslant 0.05$ except for $d_{A C}=1\left(D_{A s}=D_{A C}\right)$;

$$
\begin{aligned}
& -d_{B C} \approx d_{C s} \gg d_{B s}\left(D_{B C} \approx D_{C s} \ll D_{B s}\right) \text { for all } x_{A i} \text { and } \\
& x_{B b} ; \\
& -d_{B C} \approx d_{C s} \ll d_{B s}\left(D_{B C} \approx D_{C s} \gg D_{B s}\right) \text { and } x_{A i} \ll x_{B b} ; \\
& -x_{A i} \geqslant 0.5 \text { and } x_{B b} \geqslant 0.5 \text { and } d_{A C}, d_{B C}, d_{B s}, d_{C s} \\
& \left(D_{A C}, D_{B C}, D_{B s}, D_{C s}\right) \text { deviating significantly from } \\
& 1\left(D_{A s}\right) .
\end{aligned}
$$

Table 3. Parameter values used to calculate the data points shown in Fig. 5 for mass transfer with instantaneous chemical reaction

$$
\begin{gathered}
x_{A i} \in\{0.001,0.1,0.5\} \\
x_{B b} \in\{0.001,0.1,0.5\} \\
d_{A C} \in\{0.2,5\} \\
d_{B C} \in\{0.2,5\} \\
d_{B s} \in\{0.2,5\} \\
d_{C s} \in\{0.2,5\}
\end{gathered}
$$



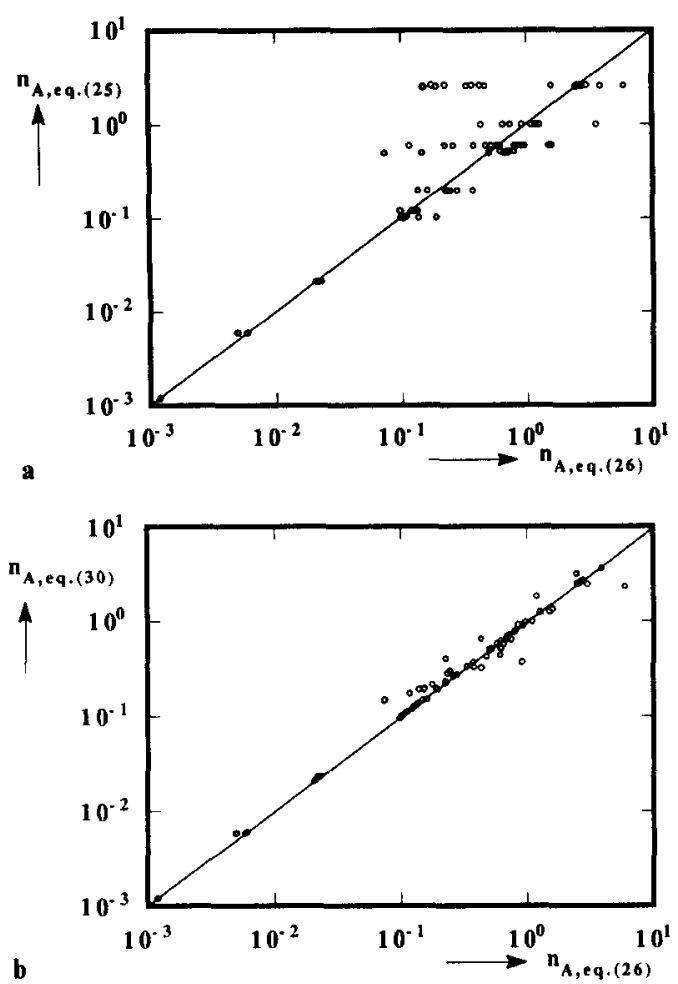

Fig. 5. Comparison of (a) Fick's law, eq. (25), and (b) an approximate explicit expression, eq. (30), with the results from the analytical solution, eq. (26). Parameter values are given in Table 3.

\subsection{Mass transfer with first-order chemical reaction}

In this case a pure gas is assumed to absorb in a solvent containing a chemically inert component $\mathbf{B}$, where the following chemical reaction takes place in the solvent:

$$
\mathrm{A} \rightarrow \mathrm{C}
$$

which is assumed to obey first-order kinetics. The solvent, component $B$ and product $C$ are not volatile and the fractions of $A$ and $C$ in the liquid bulk equal zero. In the previous section an approximate explicit expression [eq. (24)] has been derived for physical absorption of $\mathrm{A}$ in the solvent containing a chemically inert component B. Subsequently, the development of an expression for the absorption flux of $A$ will be considered in case the aforementioned chemical reaction takes place. In this development the classical theory, based on Fick's law [see e.g. Westerterp et al., (1990)], of chemically enhanced absorption will serve as the starting point. The results of the numerical calculations will be compared with the results obtained on basis of this theory.

In case of mass transfer accompanied by chemical reactions in gas-liquid systems the mass flux of the absorbed component is usually formulated as the product of an enhancement factor $E_{A}$ and the mass flux corresponding to physical absorption at the same bulk concentration:

$$
n_{A, \text { with reaction }}=E_{A} n_{A, \text { without reaction }}
$$

in which the enhancement factor $E_{A}$ is expressed by

$$
E_{A}=\frac{H a}{\tanh (H a)}
$$

where $\mathrm{Ha}$ is the Hatta number:

$$
H a=\sqrt{\frac{k_{1} \delta_{m}}{c_{T} K_{A \text { eff }}}}
$$

and $K_{A \text { eff }}$ the effective mass transfer coefficient of $A$ in the liquid mixture:

$$
\frac{1}{K_{A \text { eff }}}=\frac{x_{B b}}{K_{A B}}+\frac{1-x_{B b}}{K_{A s}}
$$

The expression for the enhancement factor $E_{A}$, eq. (32), is strictly valid in case Fick's law is used for the description of the mass transfer process. In this study it will be investigated whether the same equation can be applied in case the Maxwell-Stefan theory is used to describe the mass transfer process. To evaluate the Hatta number, an effective mass transfer coefficient of $A$ in the liquid mixture is required. Expression (34) has been derived from eqs (21) and (24) (see Appendix C). The dimensionless mass transfer flux $n_{A}$ in the absence of chemical reaction is given by eq. (24). Figure 6(a) shows a comparison between the numerically computed absorption flux and the absorption flux obtained from expression (31), using eqs (24) and (32)-(34) in case of equal mass transfer coefficients (see Table 4). From this figure it can be concluded that absorption without reaction can be described well with eq. (24). In addition, it can be concluded that the classical expressions for the enhancement factor [eq. (32)] can also be used in case the Maxwell-Stefan equations are used to describe the mass transfer process. Figures $6(\mathrm{~b})$ and $6(\mathrm{c})$ show a comparison between the numerically computed absorption flux and the absorption flux obtained form expression (31), using eqs (24) and (32)-(34) in case the mass transfer coefficients differ (see Table 4). From these figures it can be concluded that for systems with different binary mass transfer coefficients, absorption without reaction can be described well with eq. (24). Furthermore, it can be concluded that eq. (32) can be used to estimate the enhancement factor and additionally that the use of $K_{A \text { eff }}$ in the Hatta number appears to produce satisfactory results. The large error $(65 \%)$ in Fig. 6(c) for a reaction rate constant of $1 \times 10^{7} \mathrm{~mol} / \mathrm{m}^{3} \mathrm{~s}$ is due to the fact that the film composition differs significantly from the bulk composition. In this case a substantial amount of $\mathrm{C}$ has been produced in the film. Due to the fact that $K_{A C}$ is comparatively low, the effective transport coefficient of $A$ in the liquid film is also low. Equation (34) does not account for this phenomenon as it is based on bulk composition. The approximate analytical expression will predict wrong values for the mass transfer flux in case of high reaction rate constants and $K_{A C}$ values which differ significantly from $K_{A B}$ and $K_{A s}$. 

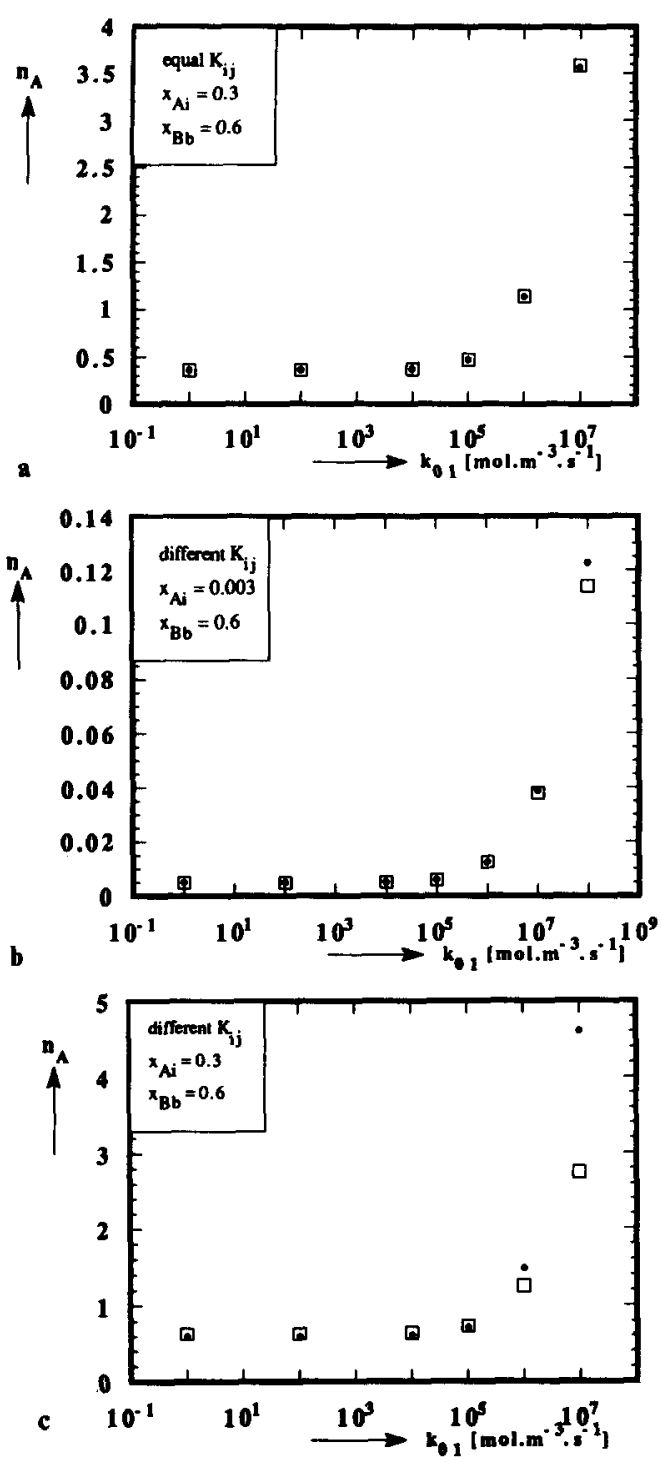

Fig. 6. Comparison of the computed numerical results ( $\square$ ) with the results obtained from the approximate analytical expression $(\cdot)$, given by eqs (24) and (31)-(34) for mass transfer with first-order chemical reaction, vs reaction rate constant. Parameter values are given in Table 4.

\subsection{Mass transfer with $(1,1)$-order chemical reaction-intermediate reaction rates}

In this case a pure gas is assumed to absorb in a solvent containing a reactive component $B$ where the following chemical reaction takes place in the solvent:

$$
\mathrm{A}+\mathrm{B} \rightarrow \mathrm{C}
$$

which is assumed to obey $(1,1)$-order kinetics. The solvent, component $B$ and product $C$ are not volatile and the fractions of $\mathrm{A}$ and $\mathrm{C}$ in the liquid bulk equal zero. In a previous section an approximate explicit expression [eq. (24)] has been derived for physical absorption of $A$ in a solvent containing a chemically inert component $\mathrm{B}$. In addition, an approximate explicit expression [eq. (30)] has been derived in case an instantaneous bimolecular chemical reaction takes place in the liquid phase. Subsequently, an expression for the absorption flux of $A$ will be developed in case the chemical reaction proceeds at finite rate. Similar to the first-order chemical reaction, eq. (31) is valid where the expression for the enhancement factor is now given by

$$
E_{A}=\frac{H a \sqrt{\frac{E_{A \infty}-E_{A}}{E_{A \infty}-1}}}{\tanh \left(H a \sqrt{\frac{E_{A \infty}-E_{A}}{E_{A \infty}-1}}\right)}
$$

where the Hatta number $H a$ is given by

$$
H a=\sqrt{\frac{k_{1} x_{B b} \delta_{m}}{c_{T} K_{A \text { eff }}}}
$$

and the maximum enhancement factor $E_{A \infty}$ is given by

$$
E_{A \infty}=\frac{n_{A, \text { with instantaneous reaction }}}{n_{A, \text { without reaction }}}
$$

The expression for the enhancement factor $E_{A}$, eq. (35), has first been derived by van Krevelen and Hoftijzer in 1948. These authors used Fick's law for the description of the mass transfer process and approximated the concentration profile of component $\mathbf{B}$ by a constant $x_{B i}$ over the entire reaction zone. It seems worthwhile to investigate whether the same equation can be applied in case the Maxwell-Stefan theory is used to describe the mass transfer process. To evaluate the Hatta number, again an effective mass transfer coefficient $K_{A \text { eff }}$, given by eq. (34), is required. The dimensionless mass transfer flux $n_{A}$ in absence of chemical reaction and in case of an instantaneous reaction are given by, respectively, eqs (24) and (30).

Table 4. Parameter values used to calculate the data points shown in Figs 6 and 7 for mass transfer with, respectively, first-order chemical reaction and $(1,1)$-order chemical reaction

\begin{tabular}{lc}
\hline$\delta_{m}=1 \times 10^{-5} \mathrm{~m}$ & Equal binary mass transfer coefficients \\
$\mathcal{c}_{T}=1 \times 10^{4} \mathrm{~mol} / \mathrm{m}^{3}$ & $K_{i j}^{l}=1 \times 10^{-4} \mathrm{~m} / \mathrm{s}, \quad j=A, B, C, s \quad i=A, B, C, s$, \\
& Different binary mass transfer coefficients \\
$H_{0 i}=0 \mathrm{~J} / \mathrm{mol}$ & $K_{A B}^{l}=3 \times 10^{-4} \mathrm{~m} / \mathrm{s}, K_{A C}^{l}=3 \times 10^{-5} \mathrm{~m} / \mathrm{s}$, \\
$C_{p t}=0 \mathrm{~J} / \mathrm{mol} K$ & $K_{A s}^{l}=1 \times 10^{-4} \mathrm{~m} / \mathrm{s}$ \\
$E_{a 1}=0 \mathrm{~J} / \mathrm{mol}$ & $K_{b C}^{l}=4 \times 10^{-4} \mathrm{~m} / \mathrm{s}, K_{B s}^{l}=5 \times 10^{-5} \mathrm{~m} / \mathrm{s}$, \\
& $K_{C s}^{l}=2 \times 10^{-4} \mathrm{~m} / \mathrm{s}$ \\
\hline
\end{tabular}


Figures $7(a)-(c)$ show a comparison between the numerically computed absorption flux and the absorption flux obtained from expression (31), using eqs (24), (30) and (34) (37). From these figures it can be concluded that for both equal and different binary mass transfer coefficients absorption without reaction can be described well with eq. (24), whereas absorption with instantaneous reaction can be described well with eq. (30). If the Maxwell-Stefan theory is used to describe the mass transfer process, the enhancement factor obeys the same expression as the one obtained on the basis of Fick's law [eq. (35)]. Finally, from Figs 7 (b) and $7(\mathrm{c})$ it appears that the use of an effective mass transfer coefficient $K_{A \text { eff }}$ in the Hatta number again produces satisfactory results.
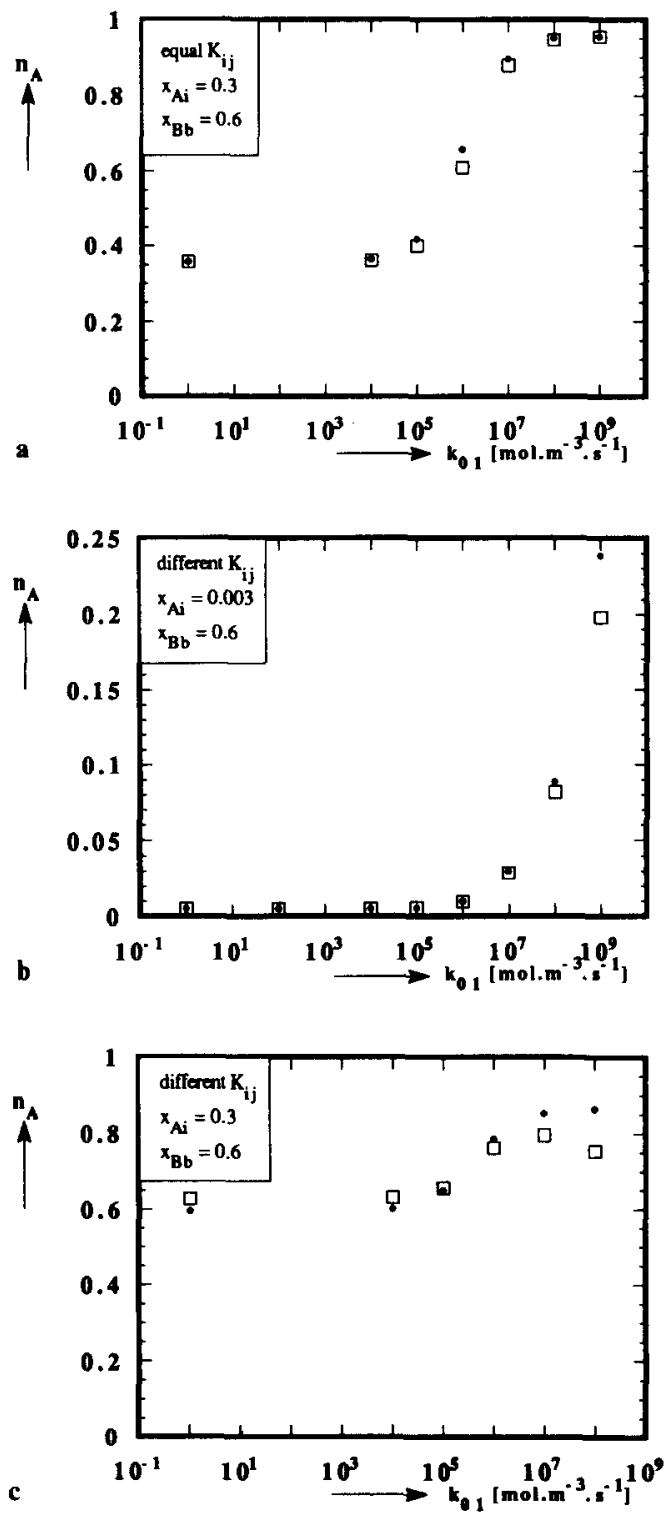

Fig. 7. Comparison of the computed numerical results ( $\square$ with the results obtained from the approximate analytical expression (-), given by eqs (24), (30), (31) and (34)-(37) for mass transfer with $(1,1)$-order chemical reaction, vs reaction rate constant. Parameter values are given in Table 4.

\subsection{Mass transfer with $(1,1)$-order chemical reaction-special case}

A closer examination of the results obtained for run 12 from Table $2\left(K_{A B}=2 \times 10^{-4} \mathrm{~m} / \mathrm{s}\right)$ revealed that an increasing reaction rate constant produced a decreasing dimensionless mass flux $n_{A}$. This surprising effect has been studied in more detail by calculating $n_{A}$ as a function of the reaction rate constant where $K_{A B}$ has been taken equal to $1 \times 10^{-3} \mathrm{~m} / \mathrm{s}$ in order to enlarge the computed effects. The results of the calculations are shown in Fig. 8. In this figure three lines are shown which correspond to the following reaction kinetics:

$$
\begin{aligned}
& R=k_{0,0} \\
& R=k_{1,0} x_{A} \\
& R=k_{1,1} x_{A} x_{B} .
\end{aligned}
$$

From this figure it can be seen that the dimensionless mass flux passes through a minimum and maximum with increasing value of the reaction rate constant where the loci of these minima and maxima depend on the reaction kinetics. The shape of the curve can be explained as follows: in case of an increasing chemical reaction rate the average fraction of $B$ in the mass transfer film will decrease. This will lead to a lower effective diffusion coefficient of $\mathbf{A}$ in the mixture, as $K_{A s}$ is smaller than $K_{A B}$, and as a consequence the fiux of $A$ will decrease. By increasing the rate constant further, the increase due to higher reaction rates will exceed the decrease due to a smaller effective diffusion coefficient and the flux will increase correspondingly. At very high reaction rates the effect of the lower effective diffusion coefficient of $\mathbf{A}$ in the mixture is more important than the effect of increased reaction rate constant and the flux decreases to the limit corresponding to an instantaneous reaction. The minimum and maximum are thus explained by the change of the effective mass transfer coefficients of components $\mathrm{A}$ and $\mathrm{B}$ with increasing $k_{m, n}$, which is only possible with a set of different binary mass transfer coefficients. It should be noted that the effects shown

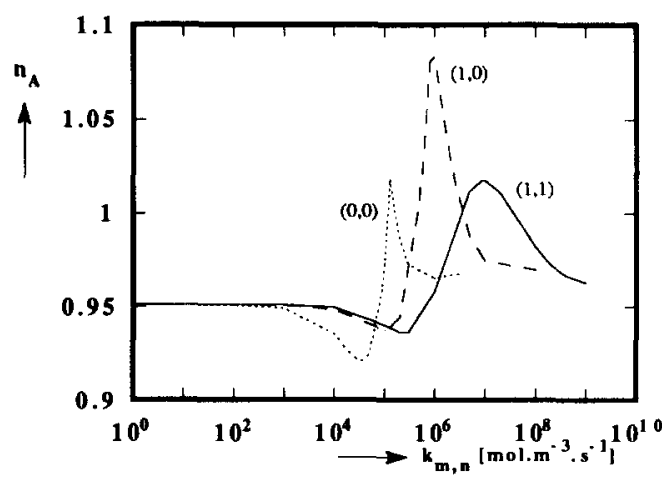

Fig. 8. Dimensionless absorption flux $n$ as function of reaction rate constant for mass transfer with bimolecular chemical reaction in case all $K_{i j}$ equal $1 \times 10^{-4} \mathrm{~m} / \mathrm{s}$ except $K_{A B}$ $\left(K_{A B}=1 \times 10^{-3} \mathrm{~m} / \mathrm{s}\right)$. The kinetics are given by eqs (38a)-(38c). 
in Fig. 8 could be demonstrated due to the fact that the maximum enhancement factor was very close to one and therefore will be of no importance for most gas-liquid absorption systems.

\subsection{Mass transfer with reversible chemical reaction}

In this case a vapour-liquid system, where high concentrations occur and all components are volatile, will be considered. The liquid and vapour bulk are not in physical equilibrium. In the liquid phase the following reversible chemical reaction occurs:

$$
\mathrm{A}+\mathrm{B} \leftrightarrow \mathrm{C}+\mathrm{D}
$$

where the reaction kinetics is given by

$$
R=k_{01} x_{A} x_{B}-k_{02} x_{C} x_{D} .
$$

The liquid bulk is assumed to be at chemical equilibrium. Contrary to gas-liquid systems, for vapour-liquid systems it is not possible to derive explicit analytical expressions for the mass fluxes which is due to the fact that two or more physical equilibrium constants $m_{i}$ have to be dealt with. This will lead to coupling of all the mass fluxes at the vapour - liquid interface since eqs (15c) and (19) have to be satisfied. For the system described above several simulations have been performed in which the chemical equilibrium constant $K=k_{01} / k_{02}$ and the reaction rate constant $k_{01}$ have been varied. Parameter values used in the simulations are given in Table 5. The results are presented in Figs 9 and 10.

Due to chemical conversion in the liquid-phase mass transfer film the mass fiux of $A$ at the vapour-liquid interface and the mass flux of $A$ at the boundary between this film and the liquid bulk will differ. Figures 9(a) and (b) show the values of these fluxes as a function of the reaction rate constant $k_{01}$ for equilibrium constants $K=1$ and $K=100$. The difference between inlet (i.e. at the vapour-liquid interface) and outlet (i.e. at the boundary between mass transfer film and liquid bulk) flux in the liquid film can be related to the amount that has been converted or produced chemically per unit time. This latter quantity is shown in Figs 10(a) and (b) as a function of the chemical reaction rate constant $k_{01}$. In case of equal binary mass transfer coefficients no maxima occur and the curves behave as we expect them to behave: an increase in equilibrium constant results in an increase in chemical conversion rate [see Fig. 10(a)]. Figure 10(b) shows that in case of different binary mass transfer coefficients this is not valid anymore. This is possibly due to variations in interaction forces as these are now dependent on component concentrations and therefore on chemical conversion.

\section{DISCUSSION AND CONCLUSIONS}

In this study a general applicable model has been developed which can predict mass and heat transfer fluxes through a vapour/gas-liquid interface in case a chemical reaction occurs in the liquid phase. In this model the Maxwell-Stefan theory has been used to describe the transport of mass and heat. A film model has been adopted which postulates the existence of a well-mixed bulk and stagnant zones where the principal mass and heat transfer resistances are situated. Due to the mathematical complexity the equations have been solved numerically by a finite-difference technique. In this paper (Part I) the Maxwell--Stefan theory has been compared with the classical theory due to Fick for isothermal absorption of a pure gas $\mathrm{A}$ in a solvent containing component $\mathrm{B}$. Component $A$ is allowed to react by a unimolecular chemical reaction or by a bimolecular chemical reaction with

Table 5. Parameter values used to calculate the profiles depicted in Figs 9 and 10 for a vapour-liquid system with reversible chemical reaction

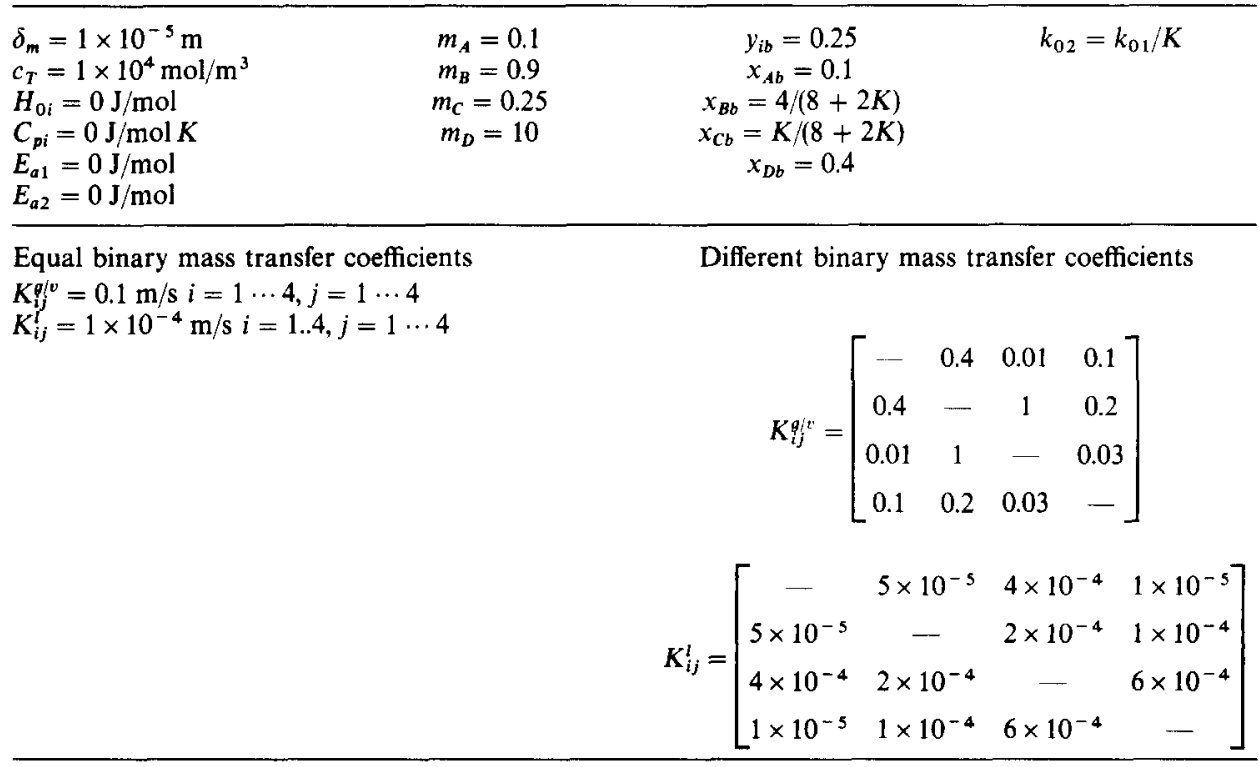



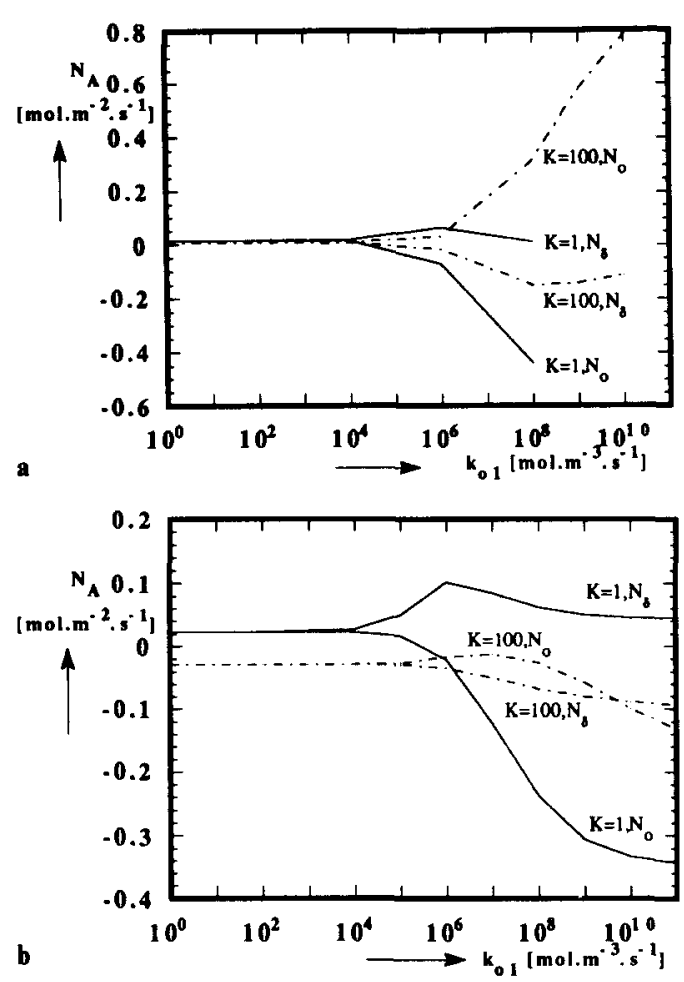

Fig. 9. The molar flux of component $A$ at the vapour-liquid interface $(\circ)$ and at the boundary between mass transfer film and liquid bulk $(\delta)$ as function of reaction rate constant in case (a) the mass transfer coefficients are equal and (b) the mass transfer coefficients are different.

component $B$ to produce component $\mathrm{C}$. When all binary diffusion coefficients are equal, mass transfer can be described by Fick's law with superimposed drift, but when the binary diffusion coefficients differ significantly, the Maxwell-Stefan flux equation should be used to account properly for all binary interactions. As the Maxwell-Stefan equation results in implicit expressions for the absorption fluxes, in case of both absorption without chemical reaction and absorption with an instantaneous bimolecular chemical reaction, approximate explicit expressions have been derived, which appear to be very succesful; respectively, eqs (24) and (30). In case of absorption with chemical reaction it turned out that the mass transfer rate could be described by the mass transfer rate without chemical reaction and an enhancement factor [eq. (31)]. This enhancement factor possesses the same functional dependency with respect to the Hatta number as is the case when Fick's law is used to describe the diffusional transport. For a vapour-liquid system, where high concentrations occur and all components are volatile, one should always use the Maxwell-Stefan theory. As all flux equations are coupled due to vapour-liquid equilibrium conditions no explicit analytical expressions can be derived. In this case numerical solution procedures are required.

The model which has been developed in this paper is very general and can be used in many gas-liquid
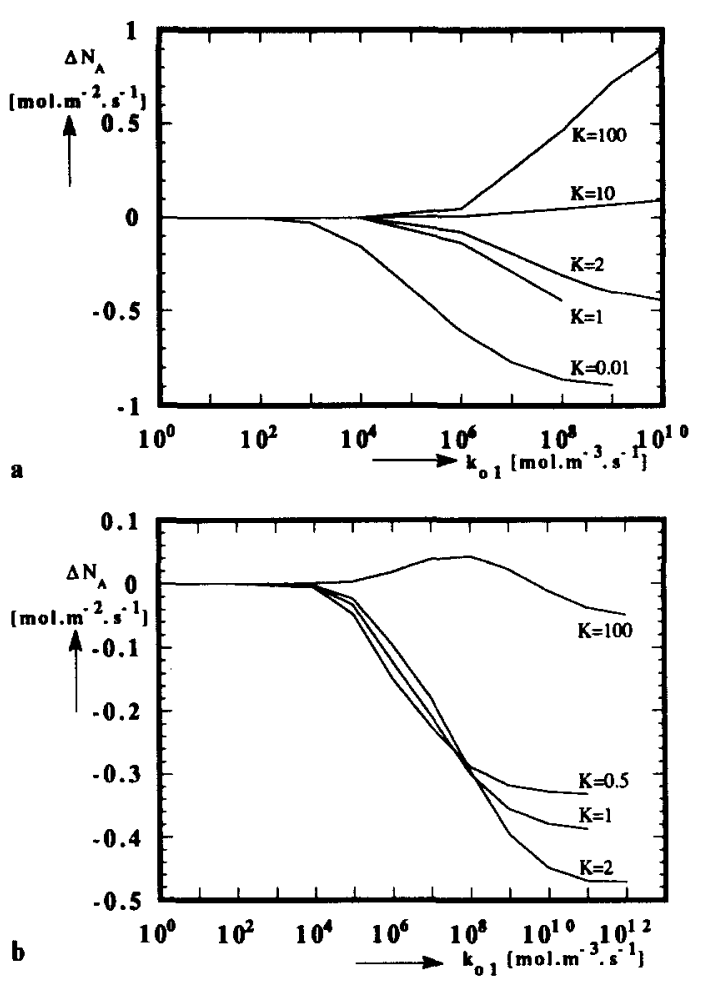

Fig. 10. The amount of component $A$ which is converted (positive) or produced (negative) chemically in the liquid film as function of the reaction rate constant in case (a) the mass transfer coefficients are equal and (b) the mass transfer coefficients are different.

and vapour-liquid transfer processes. Especially in case of reactive distillation, where mass transfer, heat transfer and chemical reaction are coupled and where high component fractions occur, the developed flux model will be very useful.

In this paper only isothermal simulations have been conducted to show the important features of the model to describe mass transfer with chemical reaction. In many industrial processes, distillation, reactive distillation and some absorption processes, heat effects play an important role and therefore cannot be neglected. These effects will be discussed in Part II.

Acknowledgements- These investigations were supported by the Foundation for Chemical Research in the Netherlands (S.O.N.). We also acknowledge R. Krishna for taking part in the discussions.

\section{NOTATION}

$c_{i} \quad$ concentration of component $i, \mathrm{~mol} / \mathrm{m}^{3}$

$c_{T} \quad$ total concentration, $\mathrm{mol} / \mathrm{m}^{3}$

$C_{p i} \quad$ heat capacity of component $i, \mathrm{~J} / \mathrm{mol} \mathrm{K}$

$d_{i j} \quad\left(=D_{a s} / D_{i j}\right)$

$D_{i j} \quad$ binary diffusion coefficient, $\mathrm{m}^{2} / \mathrm{s}$

$E$ energy flux, $\mathrm{J} / \mathrm{m}^{2} \mathrm{~s}$

$E_{A} \quad$ enhancement factor

$E_{a 1} \quad$ activation energy forward reaction, $\mathrm{J} / \mathrm{mol}$

$E_{a 2}$ activation energy backward reaction, $\mathrm{J} / \mathrm{mol}$

$E_{c} \quad$ conductive energy flux in heat transfer film from $\eta=1$ to $\eta=\mathrm{Le}^{\prime}$ 
$f \quad$ relative position of reaction plane in mass transfer film according to Fig. B1

$h \quad$ heat transfer coefficient $\left(=\lambda / \delta_{h}\right), \mathrm{J} / \mathrm{K} \mathrm{s}$

$H_{i}$ partial molar enthalpy of component $i$, $\mathrm{J} / \mathrm{mol}$

$\mathrm{Ha} \quad$ Hatta number

$k_{01}$ forward reaction rate constant, $\mathrm{mol} / \mathrm{m}^{3} \mathrm{~s}$

$k_{02}$ backward reaction rate constant, $\mathrm{mol} / \mathrm{m}^{3} \mathrm{~s}$

$K_{i j} \quad$ binary mass transfer coefficient $\left(=D_{i j} / \delta_{m}\right)$, $m / s$

$K \quad$ chemical equilibrium constant

$L e^{\prime} \quad$ modified Lewis number $\left(=\delta_{h, l} / \delta_{m, l}\right)$

$m_{i} \quad$ solubility of component $i\left(=x_{i} / y_{i}\right)$

$n_{A} \quad\left(=N_{A} / K_{A s} c_{T}\right)$

$N_{i} \quad$ molar flux of component $i, \mathrm{~mol} / \mathrm{m}^{2} \mathrm{~s}$

$p \quad$ pressure, $\mathrm{N} / \mathrm{m}^{2}$

$p_{g / v, i} \quad$ gas-vapour pressure of component $i, \mathrm{~N} / \mathrm{m}^{2}$

$q \quad$ conductive heat flux, $\mathrm{J} / \mathrm{m}^{2} \mathrm{~s}$

$R_{\text {gas }} \quad$ gas constant, $\mathrm{J} / \mathrm{mol} \mathrm{K}$

$R$ reaction rate, $\mathrm{mol} / \mathrm{m}^{3} \mathrm{~s}$

$T$ temperature, $\mathrm{K}$

$u_{i} \quad$ velocity of component $i, \mathrm{~m} / \mathrm{s}$

$x_{i}$

molar fraction (in liquid phase) of component $i$

$x_{i i}$

molar fraction (in liquid phase) of component $i$ at interface

$x_{i b} \quad$ molar fraction (in liquid phase) of component $i$ in bulk

$y_{i} \quad$ molar fraction of component $i$ in gasvapour phase

$z$

distance in film, $m$

\section{Greek letters}

$\alpha_{i j} \quad$ binary thermal diffusion factor

$\delta_{h} \quad$ thickness of heat transfer film, $m$

$\delta_{m} \quad$ thickness of mass transfer film, $m$

$\eta \quad$ distance in film $\left(=z / \delta_{m}\right)$

$\lambda$ conductivity, $\mathrm{J} / \mathrm{m} \mathrm{K} \mathrm{s}$

$\phi_{i} \quad$ volume fraction of component $i$

$\mu_{i} \quad$ chemical potential of component $i, \mathrm{~J} / \mathrm{mol}$

$v_{i} \quad$ stoichiometric coefficient of component $i$ (reactant negative, product positive)

$\omega_{i} \quad$ weight fraction of component $i$

\section{Subscripts}

A component A

b bulk

$B$ component B

C component $\mathrm{C}$

$g / v \quad$ gas-vapour phase

$i$ interface

$i \quad$ component $i$

$l \quad$ liquid phase

s solvent

\section{REFERENCES}

Agreda, V. H., Partin, L. R. and Heise, W. H., 1990, Highpurity methyl acetate via reactive distillation. Chem. Engng Prog. 2, 40-46.

Al-Ubaidi, B. H., Selim, M. S. and Shaikh, A. A., 1990, Nonisothermal gas absorption accompanied by a secondorder irreversible reaction. A.I.Ch.E. J. 36, 141-146.
Baratella, P., Carrà, S., Giardini, G. and Santi, R. S., 1974, Multistage distillation with chemical reaction. Ing. Chim. Ital. 10, 1-6.

Bhattacharya, A., Gholap, R. V. and Chaudhari, R. V., 1987, Gas absorption with exothermic bimolecular (1,1 order) reaction, A.I.Ch.E. J. 33, 1507-1513.

Carrà S., Morbidelli, M., Santacesaria, E. and Buzzi, G., 1979, Synthesis of propylene oxide from propylene chlorohydrins - II. Chem. Engng Sci. 34, 1133-1140.

Chatterjee, S. G. and Altwicker, E. R., 1987, Film and penetration theories for a first-order reaction in exothermic gas absorption. Can. J. Chem. Engng 65, 454-461.

Cieutat, D., 1989, La distillation réactive: la technologie et ses applications. Pétrole et Techn. 350, 36-40.

Corrigan, T. E. and Miller, J. H., 1968, Effect of distillation on a chemical reaction. Ind. Engng Chem. Process Des. Dev. 7, 383-384.

van Krevelen, D. W. and Hoftijzer, P. J., 1948, Kinetics of gas-liquid reactions. Rec. Trav. Chim. Pays Bas 67, 563-599.

Mann, R. and Moyes, H., 1977, Exothermic gas absorption with chemical reaction. A.I.Ch.E. J. 23, 17-23.

Mikitenko, P., 1986, La distillation réactive: principe, applications et perspectives. Pétrole et Technol. 329, 34-38.

Nelson, P. A., 1971, Countercurrent equilibrium stage separation with reaction. A.I.Ch.E. J. 17, 1043-1049.

Sawistowski, H., 1983, Distillation with chemical reaction. Nato Asi Series Ser. E 72, 391-414.

Sawistowski, H. and Pilavakis, P. A., 1979, Distillation with chemical reaction in a packed column. I. Chem. E. Symp. Series 56, 4.2/49-63.

Sivasubramanian, M. S. and Boston, J. F., 1990, The heat and mass transfer rate-based approach for modelling multicomponent separation processes, in Computer Applications in Chemical Engineering, pp. 331-336. Elsevier, Amsterdam.

Taylor, R. and Krishna, R., 1993, Multicomponent Mass Transfer. Wiley, New York.

Vanni, M. and Baldi, G., 1991, Mass transfer and chemical reaction with multicomponent diffusion. Chem. Engng Sci. 46, 2465-2472.

Venkataraman, S., Chan, W. K. and Boston, J. F., 1990, Reactive distillation using Aspen Plus. Chem. Engng Prog. 8, 45-54.

Westerterp, K. R., van Swaaij, W. P. M. and Beenackers, A. A. C. M., 1990, Chemical Reactor Design and Operation. Wiley, Chichester.

\section{APPENDIX A: MASS TRANSFER WITHOUT CHEMICAL REACTION}

\section{A.1. Algebraic derivation of eq. (22)}

For the Maxwell-Stefan theory the following set of differential equations with associated boundary conditions have to be solved:

$$
\begin{gathered}
\frac{\mathrm{d} x_{A}}{\mathrm{~d} \eta}=\frac{x_{A} N_{B}-x_{B} N_{A}}{c_{T} K_{A B}}+\frac{x_{A} N_{s}-x_{s} N_{A}}{c_{T} K_{A s}} \\
\frac{\mathrm{d} x_{B}}{\mathrm{~d} \eta}=\frac{x_{B} N_{A}-x_{A} N_{B}}{c_{T} K_{A B}}+\frac{x_{B} N_{s}-x_{s} N_{B}}{c_{T} K_{B s}} \\
\eta=0, \quad x_{A}=x_{A i} \\
\eta=1 \quad x_{A}=0 \text { and } x_{B}=x_{B b} .
\end{gathered}
$$

Solution of eq. (A2) with $N_{B}=N_{s}=0$, as both components are non-volatile, and constant $N_{A}$, as no chemical reaction occurs, with boundary condition (A4) gives

$$
x_{B}=x_{B b} \exp \left[\frac{N_{A}}{c_{T} K_{A B}}(\eta-1)\right] \text {. }
$$


Integration of eq. (A1) with $N_{B}=N_{s}=0, x_{s}=1-x_{A}-x_{B}$ after substitution of eq. (A5) for $x_{B}$ yields the following general solution for the molar fraction of component $A$ :

$$
x_{A}=1+\beta \exp \left(n_{A} \eta\right)-x_{B b} \exp \left[n_{A} d_{A B}(\eta-1)\right]
$$

with $\beta$ an integration constant which can be obtained from boundary condition (A3). Substitution of the resulting expression for $\beta$ in eq. (A6) yields after application of boundary condition (A4) the final eq. (22).

\section{A.2. Derivation of eq. (23)}

Assuming $n_{A} \ll 1$ and $d_{A B} n_{A} \ll 1$ (thus also $\left.\left|n_{A}\left(1-d_{A B}\right)\right| \ll 1\right)$, the terms $\exp \left(n_{A}\right)$ and $\exp \left[n_{A}\left(1-d_{A B}\right)\right]$ may be approximated by the following first-order linearization:

$$
f(a+b)=f(a)+b f^{\prime}(a) .
$$

Taking $a=0$ and $b$, respectively, $n_{A}$ and $n_{A}\left(1-d_{A B}\right)$ results in the following expressions:

$$
\begin{aligned}
\exp \left(n_{A}\right) & =1+n_{A} \\
\exp \left[n_{A}\left(1-d_{A B}\right)\right] & =1+n_{A}\left(1-d_{A B}\right) .
\end{aligned}
$$

Substitution of eqs (A8) and (A9) in the exact implicit expression (22) and rearranging the resulting equation gives the approximate explicit expression (23).

\section{APPENDIX B: MASS TRANSFER WITH INSTANTANEOUS CHEMICAL REACTION}

B.1. Algebraic derivation of eq. (26)

For mass transfer with instantaneous chemical reaction the concentration profiles as schematically represented in Fig. B1 are assumed where the chemical reaction only takes place at plane f. Mass transfer is described by eq. (4) with $i=\mathrm{A}, \mathrm{B}, \mathrm{C}$ and s. At the left side of plane $f, N_{B}=x_{B}=0$ (no B can pass plane $f$ ) and $N_{C}=N_{s}=0$ (product and solvent are non-volatile). This results in the following differential equations and associated boundary conditions:

$$
\begin{gathered}
\frac{\mathrm{d} x_{A}}{\mathrm{~d} \eta}=-\frac{x_{C} N_{A}}{c_{T} K_{A C}}-\frac{x_{s} N_{A}}{c_{T} K_{A s}} \\
\frac{\mathrm{d} x_{C}}{\mathrm{~d} \eta}=\frac{x_{C} N_{A}}{c_{T} K_{A C}} \\
\eta=0 \quad x_{A}=x_{A i} \\
\eta=f \quad x_{A}=0 \quad \text { and } \quad x_{C}=x_{C S} .
\end{gathered}
$$

At the right side of plane $f, N_{A}=x_{A}=0$ (no $A$ can pass plane $f$ ) and $N_{s}=0$ (solvent is non-volatile). This results in the following differential equations and associated boundary conditions:

$$
\begin{aligned}
& \frac{\mathrm{d} x_{B}}{\mathrm{~d} \eta}=\frac{x_{B} N_{C}-x_{C} N_{B}}{c_{T} K_{B C}}-\frac{x_{s} N_{B}}{c_{T} K_{B s}} \\
& \frac{\mathrm{d} x_{C}}{\mathrm{~d} \eta}=\frac{x_{C} N_{B}-x_{B} N_{C}}{c_{T} K_{B C}}-\frac{x_{s} N_{C}}{c_{T} K_{C s}} \\
& \eta=f, \quad x_{B}=0 \quad \text { and } \quad x_{C}=x_{C S} \\
& \eta=1, \quad x_{B}=x_{B b} \quad \text { and } \quad x_{C}=0 .
\end{aligned}
$$

Integration of eq. (B2) with boundary condition (B4) gives

$$
x_{C}=x_{C f} \exp \left[n_{A} d_{A C}(\eta-f)\right]
$$

Integration of eq. (B1) after substitution of $x_{S}=1-x_{A}-x_{C}$ and eq. (B9) and application of boundary conditions (B3) and (B4) gives

$$
\begin{aligned}
0= & x_{C S}\left\{\exp \left[-n_{A} f\left(d_{A C}-1\right)\right]-1\right\} \\
& +1-\left(1-x_{A i}\right) \exp \left(n_{A} f\right) .
\end{aligned}
$$

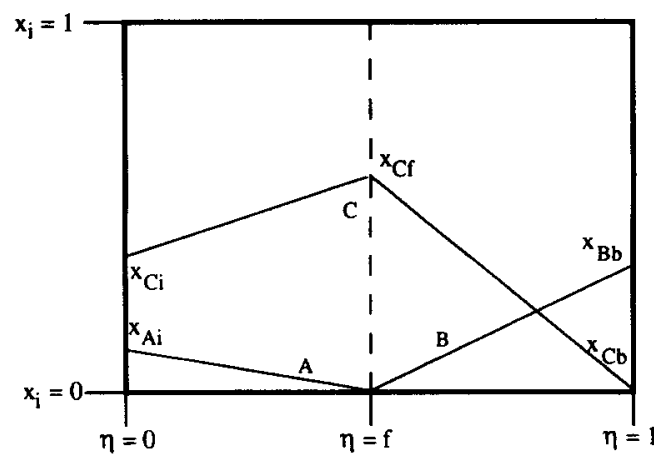

Fig. B1. Schematic representation of the fraction profiles in the liquid film in case of an instantaneous reaction $\mathrm{A}+\mathrm{B} \rightarrow \mathrm{C}$.

By adding eqs (B5) and (B6) and substituting $N_{B}=-N_{A}$ and $N_{C}=N_{A}$ (stoichiometrics) yields after application of boundary condition (B7):

$$
\begin{aligned}
& x_{B}+x_{C}=1-\left(1-x_{C S}\right) \\
& \quad \times \exp \left[n_{A}(f-\eta)\left(d_{B s}-d_{C s}\right)\right] .
\end{aligned}
$$

Applying boundary condition (B8) to eq. (B11) yields

$$
x_{B b}=1-\left(1-x_{C f}\right) \exp \left[n_{A}(f-1)\left(d_{B s}-d_{C s}\right)\right] .
$$

Now eq. (B6) can be integrated after substitution of eq. (B11) for $x_{B}$ and after application of boundary condition (B7) one obtains

$$
\begin{aligned}
0= & -n_{A} d_{B C}(1-f)-\left(1-x_{C f}\right) \frac{d_{B C}-d_{C s}}{d_{B s}-d_{C s}} \\
& \times\left\{\exp \left[n_{A}\left(d_{B s}-d_{C s}\right)(f-1)\right]-1\right\}+x_{C f}
\end{aligned}
$$

Equation (B12) gives an expression for $\left(1-x_{C f}\right)$ which can be substituted in eqs (B10) and (B13) which upon rearranging give, respectively, eqs (26a) and (26b).

\section{APPENDIX C: EFFECTIVE MASS TRANSFER COEFFICIENT}

C.1. Derivation of eq. (34)

Writing eq. (21) in parameters with dimensions gives

$$
N_{A}=-K_{A s} c_{T} \ln \left(1-x_{A i}\right)
$$

Equation (24) can be rearranged to

$$
N_{A}=-\frac{c_{T}}{\frac{1-x_{B b}}{K_{A s}}+\frac{x_{B b}}{K_{A B}}} \ln \left(1-x_{A i}\right) .
$$

From comparison of eqs $(\mathrm{C} 1)$ and $(\mathrm{C} 2)$ it can be concluded that a better expression is obtained if $K_{A s}$ is replaced by an "effective" mass transfer coefficient which takes into account the difference in binary mass transfer coefficients: eq. (34). The more general expression for the effective mass transfer coefficient of component $i$ is given by

$$
\frac{1-x_{i b}}{K_{i, \text { eff. }}}=\sum_{j} \frac{x_{j b}}{K_{i j}} .
$$

